Fundamental physics with LISA

This article has been downloaded from IOPscience. Please scroll down to see the full text article.

2009 Class. Quantum Grav. 26094020

(http://iopscience.iop.org/0264-9381/26/9/094020)

View the table of contents for this issue, or go to the journal homepage for more

Download details:

IP Address: 194.94.224.254

The article was downloaded on 09/09/2010 at 08:46

Please note that terms and conditions apply. 


\title{
Fundamental physics with LISA
}

\author{
Bernard F Schutz \\ Albert Einstein Institute, Potsdam, Germany \\ and \\ School of Physics and Astronomy, Cardiff University, Wales, UK \\ E-mail: bernard.schutz@aei.mpg.de
}

Received 16 December 2008, in final form 26 February 2009

Published 20 April 2009

Online at stacks.iop.org/CQG/26/094020

\begin{abstract}
LISA has the potential to make observations that probe deeply into fundamental physics. Not only will it test to exquisite precision the general relativity model for gravitational radiation, but will also test strong-field gravity and in particular the proposition that all black holes are described by the Kerr metric. More research is needed, however, on how to quantify the theoretical meaning of any deviations that might be seen from general relativity. LISA can also make important contributions where cosmology and fundamental physics join. LISA's observations of black-hole coalescences, out to redshifts of 20 or more, provide a completely new distance measure, one that needs no calibration and is independent of the standard cosmological 'distance ladder'. Using this measure, LISA may even begin to measure or limit the time dependence of the dark energy equation of state. Beyond these expected results from LISA is the mission's discovery space: LISA's high sensitivity raises real possibilities that it will discover sources in the dark part of the universe that were completely unexpected.
\end{abstract}

PACS numbers: 95.30.Sf, 04.30.Tv, 95.36.+x, 04.80.-y

\section{Introduction}

LISA was originally adopted by the European Space Agency (ESA) in 1995 as a Cornerstone Mission primarily in fundamental physics. Since that time there has been a huge amount of work in black hole and compact-object astronomy that has shown us how important LISA will be as an astronomical observatory. But in the same time the case for LISA as a fundamental physics mission has also considerably strengthened, especially since the discovery that the universe is accelerating. I will review the fundamental physics aspects of LISA today, occasionally pointing out work that still needs to be done in order to realize LISA's full potential when it is launched. 
I shall address four subjects: testing the theory of gravitational radiation (section 2), testing the general relativity model for strong gravitation near black holes (section 3), measuring cosmological parameters (section 4) and opening up the dark side of the universe to exploration (section 5). Readers can find more extensive reviews of some of these subjects among a soonto-appear series in the journal Living Reviews in Relativity [1, 2].

\section{Testing gravitational radiation}

As soon as LISA turns on, it should begin observing a set of known binary systems that should be strong radiators of gravitational waves, known as the verification binaries. Within a week or so, LISA could begin to make its first fundamental observation: does the radiation from these systems match expectations based on general relativity? Are there waves at all? Is the radiation frequency twice the observed orbital frequency? Is the polarization consistent with constraints on the inclination of the orbit from optical observations? While individual systems may contain surprises, the ensemble of binaries can verify that the basic quadrupole model for gravitational radiation is correct.

Given the accuracy we already have for the radiation-reaction effects in the HulseTaylor binary system [3], whose radiation lies just below the LISA frequency band, it is hardly conceivable that GR will not predict well the radiation from the verification binaries. Nevertheless, it will be reassuring actually to measure the radiation itself instead of simply observing the back-reaction on the radiating system. And as time goes on, the signal-tonoise ratio for some of the verification binary observations will get large enough to rival the accuracy of the Hulse-Taylor binary, and then LISA might begin to look for other effects, such as the scalar components predicted by some alternative gravitation theories, whose polarization would be different from that expected in general relativity.

Beyond the existence of gravitational waves, LISA can ask whether gravitational waves travel at the speed of light. Here LISA can again use the galactic binaries it observes. Of the tens of thousands of individual systems LISA will discover, some will be edge-on eclipsing systems. If some of them can be observed optically, either from the ground or from ESA's GAIA mission, then the phase of the gravitational waves can be compared with the optical orbital phase. If they are not in phase then the propagation speeds of the two forms of radiation must be different. Assuming a timing accuracy of $\pm 1 \mathrm{~min}$ for a system that is at a distance of $100 \mathrm{pc}$, LISA could test the relative speeds to parts in $10^{9}$. Much more accurate would be a bound within a model of a 'massive graviton', where dispersion of gravitational waves distorts the observed signal from an inspiralling pair of supermassive black holes [4].

Another fascinating recent suggestion [5] is that, if the gravitational Lagrangian has a Chern-Simons term, which arises in many models that try to unify gravity with known particle physics, then the resulting parity failure will cause left- and right-hand polarized waves to travel at different speeds. This would distort the observed wave forms of binary black-hole mergers, which would then be a sensitive test of string-theory-inspired gravitation.

\section{Probing strong gravitational fields}

LISA will study strong field general relativity near black holes with unprecedented accuracy. While X-ray observations are already revealing frame dragging near Kerr black holes, and while advanced LIGO and VIRGO are likely to observe many coalescing black-hole binary systems, none of these observations is likely to test GR at accuracy levels better than 5-10\% before LISA launches. By contrast, LISA will achieve signal-to-noise ratios above $10^{4}$ on 
some coalescences and will exquisitely follow the phase evolution of orbits of small bodies near massive black holes.

\subsection{Testing strong-field gravity in merging black-hole binaries}

LISA's initial observations of black-hole mergers will probably be those that create the most dramatic impression on physicists and the general public. LISA will detect each system long before the merger takes place, and will be able to give rough values of the merger time and position well in advance so that astronomers can plan to train telescopes in all wave bands on the event. On the final day the position prediction for the strongest events becomes good enough to give precise fields of view for large telescope that can take deep images. There is much to be learned about the astronomy of such systems, their initial encounter and orbits, their accretion disks, their host galaxies. But from the point of view of pure general relativity, the merger wave form itself will be a stringent test of general relativity. By comparing with accurate numerical-relativity wave forms, tests at the level of parts in $10^{3}$ or better are in prospect.

One test will be of the Cosmic Censorship Hypothesis, according to which regular initial data should not lead to naked singularities. In this case, the merger of two Kerr black holes should not produce a Kerr metric with $a / M>1$, which has a singularity but no horizon. The angular momentum available from the inspiralling orbit would be large enough to create such an object, so the system has to radiate this angular momentum away if it is to obey cosmic censorship. The signature in a merger observation would be the absence of ringdown radiation from the merger, which arises due to the trapping of gravitational waves near the horizon. Such naked singularities could also conceivably be seen as central objects for EMRI observations. The signature here would be that the EMRI signal does not disappear as the object crosses the assumed position of the horizon, but instead continues until the object encounters the singularity. It is well to bear in mind that cosmic censorship is still a hypothesis: there is no theorem in relativity to prove it. So violations are in principle possible.

Another key test from merger events would be of the Hawking area theorem. The theorem is the basis of black-hole thermodynamics, and asserts that the final black hole produced by the merger should have an area greater than the sum of the areas of the initial holes. This is a genuine theorem, but it also is deeply linked to quantum theory (through the Hawking radiation), so there is a strong motivation for testing it. However, realistic mergers seem to be far from approaching the limits of this theorem. A merger would have to be 'adiabatic' for the final black hole to have an area (entropy) just equal to the initial value. Real mergers are violent and non-adiabatic, with the result that final areas would be expected to be significantly larger than the initial areas.

\subsection{Probing strong curvature with EMRIs}

EMRIs have long provided one of the main fundamental-physics drivers of the LISA mission: to measure the geometry of a Kerr black hole in detail. EMRIs are more sensitive probes of the geometry than, for example, equal-mass black-hole mergers. This is because the inspiral chirp timescale of a binary system of a given total mass scales inversely as the mass ratio of the components, so that a system with a mass ratio of $10^{5}$, as would be typical of LISA's EMRIs, would execute something like $10^{5}$ times as many orbits while radiating in the LISA wave band as would a merging pair of equal-mass black holes. With these many orbits the signal's phase is very sensitive to the details of the geometry of the metric, and EMRI observations can be expected to show up even small deviations from the Kerr geometry. Since the Kerr geometry 
depends on just two intrinsic parameters, the mass and spin, it will not be possible to fit out deviations: it will be relatively easy to determine whether a signal comes from orbits in the Kerr metric or from some other kinds of metric. EMRI observations would therefore test the other great theorem in general relativity: black-hole uniqueness, or the fact that black holes 'have no hair'.

So far, studies of the accuracy of this test $[6,7]$ have focused on the ability of LISA to discriminate between the Kerr solution and other metric solutions of Einstein's field equations, for example metrics containing massive accretion disks [8, 9] as well as central black holes. In such situations the basic field equations are unchanged, and one simply has to determine if the observed radiation came from an orbit in one metric or another. But what if the theory is wrong? What if, for example, there is a scalar component of gravitational radiation?

The Hulse-Taylor system only weakly constrains scalar gravity because the masses of the companions are nearly equal, reducing the scalar dipole moment of the binary [10]. Black holes in such theories may radiate away their scalar field when they are formed, but an EMRI in which the smaller object is a white dwarf or neutron star will still have a scalar component and will radiate scalar waves. In this case, the scalar-tensor theory has to be well enough specified that one can calculate the radiation reaction effects on the orbiting body and compare them with the EMRI observation. This can be done if the theory is known. (See [11] and references therein.) But so far we have no framework in which to do such a calculation in a wide class of theories, and so we have no mechanism that would help us interpret a situation in which general relativity, even with an accretion disk or massive companion, significantly fails to fit the observation. This is a research problem that deserves more attention.

\subsection{EMRIs seen in binary black-hole systems}

EMRIs are compact objects (especially stellar-mass black holes) those have been deflected by stellar dynamical encounters into an orbit around the black hole that has a very small impact parameter, one where the strong radiation near pericenter passage carries away enough energy to make the system bound after a short time. Occasionally such captures should happen on one member of a supermassive black-hole binary system. If LISA observes such an EMRI, it has a chance to determine that the central black hole is accelerating toward its companion. In fact, LISA can measure this effect even for relatively wide binaries, where the companions are separated by $0.1 \mathrm{pc}$ or more.

In a three-year observation, LISA has a frequency resolution of $\Delta f=10^{-8} \mathrm{~Hz}$. At an observation frequency of $1 \mathrm{mHz}$, LISA could resolve a velocity change during the observation of $\Delta v / c \sim 10^{-5}$, which translates into an acceleration of $3 \times 10^{-5} \mathrm{~ms}^{-2}$. If both black holes have a mass of $10^{6} M_{\odot}$, then this means that they should be closer than $0.3 \mathrm{pc}$. This is in the critical 'last parsec' regime, where gravitational radiation reaction is not yet strong enough to bring the holes to coalescence in a Hubble time, but where interactions with a conventional bulge model of a galactic center may also not be strong enough to bring the holes closer together. It would be very interesting to identify galaxies that have binaries in this situation, so that they can be closely studied. Conversely, if the binary is much more compact than this, then the acceleration effects will be so strong that no template in a conventional template bank might fit the system, and the signal could be lost. Although these systems may be rare, since the range for detecting EMRIs surveys only our near neighborhood in the universe, we do not really know the number of systems that get hung up at $0.3 \mathrm{pc}$. In that sense, the higher the coalescence rate is in LISA observations, the lower will be the number of binaries containing EMRIs. For this reason the search templates for EMRIs should therefore contain an acceleration parameter to identify these systems. 


\section{Cosmogony}

We have known for 22 years that binary inspirals are standard candles [12] (standard sirens, as they are now increasingly being called [13]). If one can measure the amplitude of a chirping binary signal, even from a system with eccentricity and a high mass ratio, one can measure its luminosity distance. To measure the amplitude one has to have a good position and a measurement of polarization, which LISA can accomplish. Advanced LIGO and VIRGO can also do this with inspiralling neutron-star signals, and these observations should yield an independent measurement of the Hubble constant locally, say out to $100 \mathrm{Mpc}$ [12]. Beyond that, LISA can use measurements of EMRI events out to modest redshifts (say $z=0.5$, depending on the event rate) to get a much more accurate value for $H_{0}$ (accurate to $1 \%$ with 20 events) [14]. And with black-hole coalescences at even larger distances, LISA can measure the acceleration of the universe and thereby the present value of the 'dark energy equation of state' [15]. Even if these measurements do no more than verify the values that are already known by the time LISA flies, this will be important, because LISA's distance scale is calibration-free. It depends only on the validity of general relativity, and in particular does not need to use the complex distance ladders that astronomers have come up with to measure larger and larger distances.

However, to do this kind of cosmogony LISA has to have some way of identifying the host galaxies of the merger events, because it has to combine its chirp distances with the redshifts of the objects. The gravitational wave signal contains no redshift information. (This is not strictly true, and post-LISA missions may be able to measure intrinsic redshifts too [16].) It is possible to use statistical methods to overcome this disadvantage when the measurement errors are smaller than the errors produced by mis-identifying galaxies in the initial LISA error box: both $[12,14]$ show how effective this can be. But on a cosmological scale this may not be enough. This led the Dark Energy Task Force not to include LISA's capabilities in their review of potential ways of improving our knowledge about the dark energy:

Other techniques ..., such as using ... gravitational waves from coalescing binaries as standard candles, merit further investigation. At this time, they have not yet been practically implemented, so it is difficult to predict how they might be part of a dark energy program. We do note that if dark energy dominance is a recent cosmological phenomenon, very high redshift $(z \gg 1)$ probes will be of limited utility [17].

However, there has been much recent work examining potentially observable effects of blackhole mergers on their host galaxies (e.g. [18]), and this has been accompanied by an improved understanding of how accurate LISA's initial position error box could be, particularly when one includes the high-frequency signal components into the template [19]. Many papers in these proceedings address the latest developments in this area. Taking some of this work into account, the Beyond Einstein Program Advisory Committee took a very different view of LISA's capabilities in its report to NASA and the Department of Energy, just one year after that of the Dark Energy Task Force:

LISA also has the potential to measure the dark energy equation of state, along with the Hubble constant and other cosmological parameters. Through gravitational wave form measurements LISA can determine the luminosity distance of sources directly. If any of these sources can be detected and identified as infrared, optical or X-ray transients and if their redshift can be measured, this would revolutionize cosmography by determining the distance scale of the universe in a precise, calibration-free measurement [20]. 


\subsection{LISA as a dark-energy mission}

If LISA flies at the end of the second decade of the 21 st century, then previous missions and observing programs with ground-based telescopes might by that time have brought more clarity to the question of the dark energy. We might, in particular, have a well-determined value of the dark energy equation of state parameter $w$, defined as the proportionality between pressure $p$ and energy density $\rho$ of the 'cosmological fluid':

$$
p=w \rho .
$$

If this turns out to be -1 , the value associated with the cosmological constant, then theoretical work is likely to focus on deriving a cosmological constant of the appropriate size from fundamental physics theories. If, on the other hand, there is a measurable deviation of $w$ from -1 , then there will be a need to distinguish between many possible quintessence models.

LISA can make a contribution by extending the range of its dark-energy measurements to redshifts beyond those that can be explored by the dark energy experiments that are likely to be performed in the next 10 years. It can expect to measure coalescences out to $z=20$. As the Dark Energy Task Force report emphasized, it becomes harder and harder to measure the dark energy component as the redshift increases because the dark energy becomes a smaller and smaller fraction of the total energy density, at least if $w$ stays close to -1 . With this value of $w$, the energy density of the cosmological fluid is almost constant in time while the energy density of matter increases as $(1+z)^{-3}$. But it might happen that the dark energy equation of state evolves with redshift. We can parametrize this evolution with the following Taylor expansion in $z$ :

$$
w=-1+w_{1} z+O\left(z^{2}\right)
$$

where we assume that $w=-1$ today for simplicity, as is indicated by present measurements. If the constant $w_{1}$ is positive, then the cosmological fluid is 'causal' as the redshift increases.

The effect of this kind of cosmological fluid on the expansion rate of the universe is summarized in the following relation, which gives the Hubble parameter as a function of redshift in terms of the present fractional matter energy density $\Omega_{m}$ and dark energy density $\Omega_{\Lambda}$, ignoring the contribution of radiation, which is negligible at present:

$$
H^{2}(z)=H_{0}^{2}\left[\Omega_{m}(1+z)^{3}+\Omega_{\Lambda}(1+z)^{3 w_{1}} \mathrm{e}^{-3 z w_{1} /(1+z)}\right] .
$$

If we use concordance values for the present densities, $\Omega_{m}=0.30$ and $\Omega_{\Lambda}=0.70$, and if there is no evolution $\left(w_{1}=0\right)$, then the dark energy will drop from its present dominance of the Hubble parameter for making a contribution to $H(z)$ that is less than $30 \%$ at $z=1$, less than $9 \%$ at $z=2$ and just about $4 \%$ at $z=3$. On the other hand, if $w_{1}=0.2$, so that there is a $20 \%$ change in the dark energy equation of state parameter back to redshift 1 , then at $z=2$ the dark energy is $11 \%$ of the matter contribution, while at $z=3$ it is a little above $5 \%$.

From this it is clear that, for LISA to measure a $20 \%$ change in the equation of state, it has to be able to distinguish between $9 \%$ and $11 \%$ at $z=2$, i.e. it needs to measure the distanceredshift relationship to around $1 \%$ accuracy at this redshift. This is certainly a challenge, but it is not necessarily out of reach. The intrinsic signal-to-noise of black-hole binary events at this redshift will be many thousands, which means that the 'raw' distance measurement accuracy of LISA will be much better than $1 \%$. The biggest problem will be weak lensing, which produces random magnifications and demagnifications, which at this redshift can add up to a 5-10\% error in distance for each individual event [13].

For LISA to reach the $1 \%$ level requires either a large number of events (of order 100 during its lifetime) or some way of reducing the effect of weak lensing, such as producing lensing maps near the position of the LISA source, from observations of lensing effects on 
galaxies. This is a subject that deserves more research, considering the implications that it could have for this aspect of LISA's fundamental physics return.

\section{Unexpected discoveries}

The emergence of string theory as a viable path toward quantum gravity has led to a rich field of speculations about how quantum gravity effects might be observable at low energies, accessible to experiments in the near future. Fundamental physicists are faced by a number of challenges that have been fertile stimuli to new theoretical thinking, among which are the following.

- Unifying the strong and electromagnetic interactions into a grand unified theory.

- Showing why the universe has a non-zero baryon number (CP violation).

- Explaining why the universe is so well fine tuned for life to exist (multiverse models, the Everitt-Wheeler picture, etc).

Because we expect general relativity to give way to a quantum theory, we expect corrections at least at the Planck scale. But there are some reasons why corrections might be visible at lower energies:

- Branes. Since string theory requires 10 or 11 dimensions, there is plenty of freedom for new physical ideas. Instead of compactifying the extra dimensions, nature could have allowed one or more of them to be large. Our universe would then be a four-dimensional 'brane' embedded in a larger space. Because of the way string theory unifies gravity with the rest of physics, all of non-gravitational physics would be confined to our brane, but gravity would extend off it into the bulk. If the size of the bulk is large then measurements on gravity might show unexpected effects [21]. LISA and other gravitational experiments are the only way to 'touch' the bulk.

- Emergent gravity. It could happen that gravity is not after all a fundamental interaction, but is rather an effective theory based on some other kind of microphysics, emerging at low energies in much a way that superfluidity emerges from molecular dynamics. The Planck scale would be irrelevant and we might begin to see the transition from the effective theory to the underlying physics at much lower energies [22].

- Loops and topology. The main alternative to string theory today as a route to quantum gravity is loop quantum gravity. It may be that on short scales the apparently smooth manifold of general relativity resolves into a complex interconnected mesh of loops that have no particular dimension and a random structure. Already loop quantum gravity has predicted that it would be possible for the universe to have tunneled through the big bang from some earlier state [23].

There are already a few ideas where low-energy effects might turn up in gravitational wave observations. These include

- Cosmic strings. These could be residual defects from an earlier era of spontaneous symmetry breaking, or they might be quantum fluctuations in string theory that have been stretched into macroscopic reality by inflation [24]. See also the paper by Siemens in these proceedings.

- Stochastic gravitational wave background. LISA will be sensitive to this background at the level of $\Omega_{\mathrm{gw}} \sim 10^{-10}$. Standard slow-roll inflation predicts a smaller level, around $10^{-15}$ today. But other sources are possible, particularly in the LISA band. Any radiation 
that is emitted with wavelengths the size of the particle horizon at a time when the temperature of the universe was $T_{e}$ will have a frequency $f_{0}$ today given by

$$
f_{0}=10^{-17} \mathrm{~Hz} \frac{k T_{e}}{1 \mathrm{eV}} .
$$

The electroweak phase transition took place as the universe expanded through a temperature where $k T_{e} \sim 10^{12} \mathrm{eV}$. This radiation would be at and somewhat above $0.01 \mathrm{mHz}$ today, so that LISA would be well placed to observe this. Whether the radiation is there or not depends on whether the phase transition was first order (lots of density fluctuations) or second order (smooth). The standard electroweak theory predicts a second-order transition with very little radiation. But some theories that attempt to solve the matter-antimatter asymmetry problem within electroweak theory predict a strongly first-order transition [25].

- String theory could make many modifications in gravity, including adding in extra fields. In principle the best chance for observing such modifications of gravity is in EMRI observations, as discussed above.

- Branes. Within string theory, the brane paradigm offers many new possibilities [21]. There could be new sources of gravitational radiation from effects to do with the interaction of the brane and the bulk [26]. There could be waves in our universe generated by 'shadow matter' in a parallel brane not far from ours in the bulk [27, 28]. Or there could simply be no stochastic background radiation at all, for example as predicted for an Ekpyrotic universe [29]; but see also [30] for a way around this.

Given that $96 \%$ of the universe is dark and uncharged, it is very possible that LISA will open up an entirely new perspective on our universe by revealing structures in the dark universe that we had not imagined would be there. If LISA is to realize this potential, however, then the data analysis has to be done well enough that weak unexpected signals can be seen against the confusion caused by the strong but expected signals. This is a challenge that is being worked on intensively today [31].

\section{Conclusions}

LISA was rated by the BEPAC committee [20] as the highest priority mission in NASA's Beyond Einstein program, which was a group of missions with strong fundamental-physics aims. It is fair to conclude from this and from LISA's placement in ESA's program that LISA is the premier fundamental physics mission under development today. As with most pioneering missions, it is possible to anticipate only to a certain extent what LISA will do, but what we can anticipate is already very exciting: verifying Einstein's model for gravitational radiation, studying black holes in detail, measuring the changing rate of expansion of the universe, looking for exotica like cosmic strings and the radiation from the big bang. Once LISA flies it has the potential to completely change the way we look at the universe.

\section{References}

[1] Hogan C, Hughes S, Sintes A and van den Broeck C 2009 Cosmology with LISA Living Rev. Rel. at press (see http://www.livingreviews.org/)

[2] Baker J, Gair J, Larson S and Vallisneri M 2009 Testing general relativity with LISA Living Rev. Rel. at press (see http://www.livingreviews.org/)

[3] Weisberg J M and Taylor J H 2005 The relativistic binary pulsar B1913 + 16: thirty years of observations and analysis Binary Radio Pulsars (ASP Conf. Series vol 328) ed F A Rasio and I H Stairs p 25 (San Francisco, CA: ASP) 
[4] Will C M 1998 Bounding the mass of the graviton using gravitional-wave observations of inspiralling compact binaries Phys. Rev. D 572061

[5] Alexander S and Yunes N 2008 Chern-Simons modified gravity as a torsion theory and its interaction with fermions Phys. Rev. D 77124040

[6] Glampedakis K and Babak S 2006 Mapping spacetimes with LISA: inspiral of a test-body in a 'quasi-Kerr' field Class. Quantum Grav. 23 4167-88

[7] Barack L and Cutler C 2007 Using LISA EMRI sources to test off-Kerr deviations in the geometry of massive black holes Phys. Rev. D 75042003

[8] Barausse E, Rezzolla L, Petroff D and Ansorg M 2007 Phys. Rev. D 75064026

[9] Barausse E and Rezzolla L 2008 Phys. Rev. D 77104027

[10] Will C M 2001 The confrontation between general relativity and experiment Living Rev. Rel. 44

[11] Berti E, Buonanno A and Will C M 2005 Class. Quantum Grav. 22 S943-54

[12] Schutz B F 1986 Determining the Hubble constant from gravitational wave observations Nature 323 310-1

[13] Holz D E and Hughes S A 2005 Using gravitational-wave standard sirens Astrophys. J. 629 15-22

[14] MacLeod C L and Hogan C J 2008 Precision of Hubble constant derived using black-hole binary absolute distances and statistical redshift information Phys. Rev. D 77043512

[15] Dalal N, Holz D E, Hughes S A and Jain B 2006 Short GRB and binary black hole standard sirens as a probe of dark energy Phys. Rev. D 74063006

[16] Seto N, Kawamura S and Nakamura T 2001 Possibility of direct measurement of the acceleration of the universe using $0.1 \mathrm{~Hz}$ band laser interferometer gravitational wave antenna in space Phys. Rev. Lett. 87221103

[17] Kolb E et al 2006 Report of the dark energy task force Report to the Astronomy and Astrophysics Advisory Committee and the High Energy Physics Advisory Panel (Available at http://arxiv.org/abs/astro-ph/0609591)

[18] Milosavljević M and Phinney E S 2005 The afterglow of massive black hole coalescence Astrophys. J. Lett. 622 L93-6

[19] Babak S, Hannam M, Husa S and Schutz B 2008 Resolving super massive black holes with LISA arXiv:0806.1591

[20] Kennel C F and Rothenberg J H et al NASA's Beyond Einstein Program: An Architecture for Implementation (Washington, DC: National Academy of Sciences) (Downloaded from http://www.nap.edu/ catalog/12006.html)

[21] Maartens R 2004 Brane-world gravity Living Rev. Rel. 77

[22] Volovik G E 2003 The Universe in a Helium Droplet (Oxford: Oxford University Press)

[23] Bojowald M 2008 Loop quantum cosmology Living Rev. Rel. 114 (available at http://www.livingreviews. org/lrr-2008-4)

[24] Damour T and Vilenkin A 2005 Gravitational radiation from cosmic (super)strings: bursts, stochastic background, and observational windows Phys. Rev. D 71063510

[25] Megevand A and Astorga F 2005 Generation of baryon inhomogeneities in the electroweak phase transition Phys. Rev. D 71023502

[26] Randall L and Servant G 2007 Gravitational waves from warped spacetime J. High Energy Phys. JHEP05(2007)54

[27] Maeda K-I and Wands D 2000 Dilaton gravity on the brane Phys. Rev. D 62124009

[28] Clarkson C and Seahra S S 2007 Class. Quantum Grav. 24 F33-40

[29] Boyle L A, Steinhardt P J and Turok N 2004 The cosmic gravitational wave background in a cyclic universe Phys. Rev. D 69127302

[30] Lehners J-L, McFadden P, Turok N and Steinhardt P J 2007 Generating ekpyrotic curvature perturbations before the big bang Phys. Rev. D 76103501

[31] Babak S et al 2008 Class. Quantum Grav. 25184026 